\title{
Model-independent inference of laser intensity
}

\author{
T. G. Blackburn $\odot,{ }^{1, *}$ E. Gerstmayr $\odot,{ }^{2}$ S. P. D. Mangles $\odot,{ }^{2}$ and M. Marklund ${ }^{1}$ \\ ${ }^{1}$ Department of Physics, University of Gothenburg, SE-41296 Gothenburg, Sweden \\ ${ }^{2}$ The John Adams Institute for Accelerator Science, Imperial College London, \\ London SW7 2AZ, United Kingdom
}

(Received 6 November 2019; accepted 13 May 2020; published 11 June 2020)

\begin{abstract}
An ultrarelativistic electron beam passing through an intense laser pulse emits radiation around its direction of propagation into a characteristic angular profile. Here, we show that measurement of the variances of this profile in the planes parallel and perpendicular to the laser polarization, and the mean initial and final energies of the electron beam, allows the intensity of the laser pulse to be inferred in a way that is independent of the model of the electron dynamics. The method presented applies whether radiation reaction is important or not, and whether it is classical or quantum in nature, with an accuracy of a few percent across 3 orders of magnitude in intensity. It is tolerant of electron beams with a broad energy spread and finite divergence. In laser-electron-beam collision experiments, where spatiotemporal fluctuations cause the alignment of the beams to vary from shot to shot, this permits inference of the laser intensity at the collision point, thereby facilitating comparisons between theoretical calculations and experimental data.
\end{abstract}

DOI: 10.1103/PhysRevAccelBeams.23.064001

\section{INTRODUCTION}

Electromagnetic fields of extraordinary strength are produced at the focus of modern high-power lasers [1], inducing nonlinear classical, even quantum, dynamics of particles and plasmas [2-4]. The amplitude of these fields, expressed covariantly through the normalized vector potential $a_{0}$, plays an essential role in determining which regime is explored. However, it remains difficult to diagnose in situ the intensity reached in experiments. This is particularly acute for experiments at or beyond the current intensity frontier, which will explore the transition to the nonlinear quantum regime [5,6]. As the dynamics are not fully understood, it is important to know what $a_{0}$ is reached for comparison between competing theoretical descriptions and experimental data. Furthermore, the method by which $a_{0}$ is determined should not be sensitive to the underlying physics, particularly if the latter is the subject of the experiment.

The method presented here is based on the collision of an ultrarelativistic electron beam with the laser pulse; this geometry has been already been exploited as a high-energy photon source [7-9] and a probe of radiation reaction $[10,11]$. Measurement of the angular profile of the resulting radiation has been proposed as a means of determining the

\footnotetext{
*tom.blackburn@physics.gu.se

Published by the American Physical Society under the terms of the Creative Commons Attribution 4.0 International license. Further distribution of this work must maintain attribution to the author(s) and the published article's title, journal citation, and DOI. Funded by Bibsam.
}

peak intensity of a laser pulse $[12,13]$ (the former demonstrated experimentally in Ref. [9]), as has measurement of the electron scattering angles [14]. However, the results presented in Refs. [12-14] depend critically on the model assumed for the electron dynamics. The appropriate choice of model depends on the intensity of the laser to be probed: For example, at a very high intensity, radiation reaction and quantum effects are expected to become important, if not dominant [4]. A method that does not require such an assumption to be made would be a useful complement to methods that are model dependent, providing stronger evidence that a particular regime has been reached.

Here, we show that the laser intensity can be inferred in a model-independent way, using the angular profile of the radiation emitted in the collision of the laser with an electron beam, in combination with the mean initial and final energies of the beam. We derive analytical predictions for the size of the radiation profile and the energy change of the electron beam, treating the laser as a pulsed plane electromagnetic wave, that can be combined so as to eliminate an explicit dependence on classical radiation reaction. We show that this model-independence applies to a high degree of accuracy under quantum models of radiation reaction as well.

Examining our method in a more realistic scenario, where the tight focusing of the laser and finite size of the electron beam are taken into account, we find that it yields a model-independent estimate of the laser intensity, averaged over the electron-beam size. Furthermore, it is robust against finite energy spread and angular divergence, two important characteristics of nonideal electron beams; the latter, in particular, controls the overlap between the laser 
and electron beam. This permits measurement of the peak intensity, if the electron beam is well characterized, stable, and radially smaller than the laser focal spot size; with spatiotemporal fluctuations taken into account, our method provides a powerful constraint on the intensity at the point of interaction, for each individual collision. This is complementary to methods aimed at determining the peak intensity itself, by measurement of the ionization level of heavy atoms [15], Thomson scattering of low-energy electrons present in the focal volume [16], or detailed characterization of the laser structure, gathered over hundreds of shots [17]. In conjunction with these, our method provides a means of determining the shot-to-shot overlap between the laser pulse and electron beam.

\section{ANALYTICAL RESULTS}

Consider an electron (charge $-e$ and mass $m$ ) with Lorentz factor $\gamma \gg a_{0}$ oscillating in a linearly polarized, plane electromagnetic wave that has normalized amplitude $a_{0}$ and frequency $\omega_{0}$. Over a single cycle of the wave, the angle between the electron momentum and the laser axis is $\theta(\phi)=a_{0} \sin \phi / \gamma$, and the electron's quantum parameter $\chi(\phi)=2 \gamma a_{0} \omega_{0}|\cos \phi| / m$. Here, $\phi$ is the phase, and the angle $\theta$ lies in the plane defined by the laser's electric field and wave vector; we refer to angles in this plane as being parallel $(\|)$ to the laser polarization. We work throughout in natural units where $\hbar=c=1$. The distribution of energy radiated per unit angle $\mathrm{d} \mathcal{E}_{\text {rad }} / \mathrm{d} \theta$ may be calculated by integrating the Larmor power, which is proportional to $\chi^{2}(\phi)$, over the cycle and assuming that the radiation is strongly beamed along the electron's instantaneous momentum; i.e., at phase $\phi$, the emission angle is $\theta(\phi)$. We then normalize the result by the total radiated energy to obtain $\mathrm{d} \mathcal{E}_{\text {rad }} / \mathrm{d} \theta=2 \gamma \sqrt{1-\left(\gamma \theta / a_{0}\right)^{2}} /\left(\pi a_{0}\right)$ for $|\theta|<a_{0} / \gamma$. The variance of the distribution is $\sigma_{\|}^{2}=\int \theta^{2} \mathrm{~d} \mathcal{E}_{\text {rad }}=a_{0}^{2} /\left(4 \gamma^{2}\right)$.

To incorporate a pulse envelope $f(\phi)$ and the effect of radiation reaction into this result, we use the fact that the variances introduced each cycle may be added linearly. The contribution of each cycle of the pulse to the total variance is $a_{0}^{2} f^{2}(\phi) /\left[4 \gamma^{2}(\phi)\right]$, weighted by $\gamma^{2}(\phi) f^{2}(\phi)$. The weighting comes from the Larmor power, which is proportional to the square of the instantaneous Lorentz factor $\gamma(\phi)$ and the local electric field, which is proportional to $f(\phi) \cdot \gamma(\phi)$ is obtained by solution of the Landau-Lifshitz equation [18], which accounts for the self-consistent radiative energy loss.

The total variance, in the direction parallel to the laser polarization, can be expressed compactly in terms of the final Lorentz factor $\gamma_{f}$ and integrals over the pulse envelope:

$$
\sigma_{\|}^{2}=\frac{a_{0}^{2}}{4 \gamma_{i} \gamma_{f}} \frac{\int f^{4}(\phi) \mathrm{d} \phi}{\int f^{2}(\phi) \mathrm{d} \phi}+\sigma_{\perp}^{2} .
$$

The second term in Eq. (1) accounts for the contributions of the intrinsic divergence of the radiation and any initial divergence of the electron beam $\delta$, which we assume to be cylindrically symmetric. These are the only contributions in the direction perpendicular to the laser polarization and wave vector [19]:

$$
\sigma_{\perp}^{2}=\frac{5}{8 \gamma_{i} \gamma_{f}}+\delta^{2}
$$

In both Eqs. (1) and (2), we have [20]

$$
\gamma_{f}=\frac{\gamma_{i}}{1+R \gamma_{i}}, \quad R=\frac{2 \alpha a_{0}^{2} \omega_{0}}{3 m} \int_{-\infty}^{\infty} f^{2}(\phi) \mathrm{d} \phi,
$$

where $\alpha=e^{2} /(4 \pi)$ is the fine-structure constant. In the absence of a radiation reaction, $\alpha=0$ and $\gamma_{i}=\gamma_{f}$. If the intensity profile $f^{2}(\phi)$ is a Gaussian with full width at half maximum (FWHM) duration $\tau$, we have $\int f^{2}(\phi) \mathrm{d} \phi=$ $\omega_{0} \tau \sqrt{\pi /(4 \ln 2)} \quad$ and $\int f^{4}(\phi) \mathrm{d} \phi=\left[\int f^{2}(\phi) \mathrm{d} \phi\right] / \sqrt{2}$. Notice that the radiation profile is elongated along the polarization direction. Strictly, this result is valid for $a_{0} \gtrsim 1$, as we have in this work; otherwise, the profile would be dipolar in shape [21].

A comparison of Eqs. (1) and (2) to the results of simulations is shown in Fig. 1. In these, the plane-wave laser pulse has a Gaussian temporal envelope with FWHM duration $\tau=40 \mathrm{fs}$ and a wavelength of $0.8 \mu \mathrm{m}$. The energies of the beam electrons are normally distributed, with a mean of 500 or $1000 \mathrm{MeV}$ and standard deviation $50 \mathrm{MeV}$ in both cases; the initial divergence is $\delta=2 \mathrm{mrad}$. Three models of the dynamics are considered: no radiation reaction (RR), i.e., the Lorentz force only; classical RR in the Landau-Lifshitz prescription; and a fully stochastic, quantum model using probability rates calculated in the locally constant field approximation [22]. These models are described in detail in the Appendix.
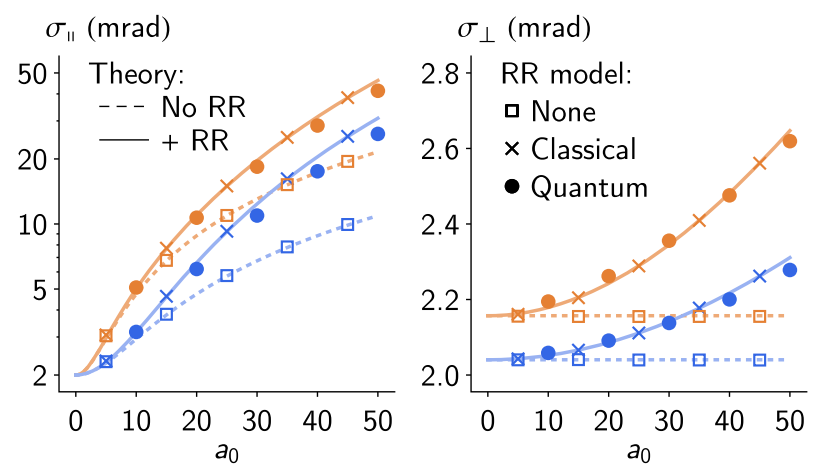

FIG. 1. Parallel and perpendicular standard deviations of the angular profile of the radiation emitted by an electron beam with mean initial energy 500 (orange) and $1000 \mathrm{MeV}$ (blue), as predicted by Eqs. (1) and (2) with (solid curves) and without RR (dashed curves) and from simulations with the specified radiation-reaction model (points). 
Radiation is generated by Monte Carlo sampling of the classical synchrotron spectrum in the former two cases and the quantum synchrotron spectrum in the latter, as is appropriate. This method is applicable at high intensity and at high harmonic order in both the classical [23,24] and quantum regimes $[25,26]$, where the photon formation length becomes much smaller than the wavelength of the driving laser [22]. Such photons dominate the power spectrum, which is the object of analysis in this work, even for relatively low $a_{0}$ [27]. We use a synchrotron spectrum that is differential in both energy and angle in our particle-tracking code, thereby resolving the intrinsic divergence of the radiation around the electron's instantaneous velocity vector $[19,28]$. Expressions for the emission spectrum are given in the Appendix for each model.

We find excellent agreement between the analytical predictions and the simulation results for classical and no RR. It is clear that the radiation reaction leads to an increased broadening of the angular profile in the plane of polarization. Furthermore, the classical and quantum models give generally similar results, even though the broadening is smaller for the latter because the radiated power is reduced. In the plane perpendicular to the laser polarization, the variance is dominated by the initial divergence of the electron beam, and radiation-reaction effects are weaker.

The effect of a finite energy spread on the variances is surprisingly small. Consider a beam of electrons, in which the initial Lorentz factors $\gamma_{i}$ are distributed as $\frac{\mathrm{d} N_{e}}{\mathrm{~d} \gamma_{i}}$. The variance of the radiation angular profile, in the direction parallel to the laser polarization, for the beam as a whole, is obtained by integrating Eq. (1), weighted by $\left(\gamma_{i}-\gamma_{f}\right) \frac{\mathrm{d} N_{e}}{\mathrm{~d} \gamma_{i}}$, over all $\gamma_{i} \gg 1$. The weighting reflects the fact that

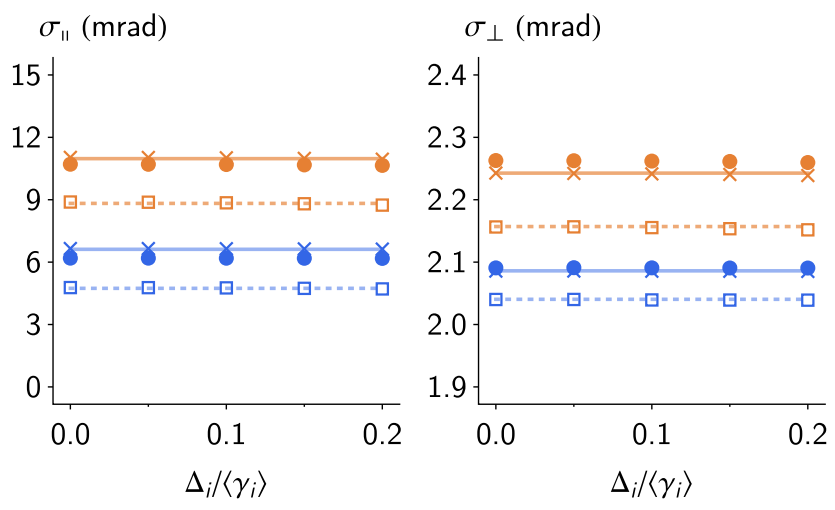

FIG. 2. Parallel and perpendicular standard deviations as a function of initial energy spread $m \Delta_{i}$, for an electron beam with mean initial energy $m\left\langle\gamma_{i}\right\rangle=500$ (orange) and $1000 \mathrm{MeV}$ (blue) colliding with a laser pulse with $a_{0}=20$ : simulation results with quantum (filled circles), classical (crosses), and no (squares) radiation reaction (as in Fig. 1) and theoretical predictions Eqs. (1) and (2) with (solid curves) and without (dashed curves) RR, assuming that the beam is monoenergetic at the mean energy. electrons with higher $\gamma_{i}$ radiate more energy and, therefore, contribute more to $\sigma_{\|}^{2}$. We obtain

$$
\sigma_{\|}^{2}=\frac{a_{0}^{2}}{4 \sqrt{2}}\left[\left\langle\gamma_{i}\right\rangle\left\langle\gamma_{f}\right\rangle+\operatorname{cov}\left(\gamma_{i}, \gamma_{f}\right)\right]^{-1}+\sigma_{\perp}^{2},
$$

where $\left\langle\gamma_{i, f}\right\rangle$ are the mean initial and final Lorentz factors, respectively. The covariance term $\operatorname{cov}\left(\gamma_{i}, \gamma_{f}\right) \leq \Delta_{i} \Delta_{f} \leq$ $\Delta_{i}^{2}$, where the $\Delta_{i, f}$ are the standard deviations of $\gamma_{i, f}$; the equality holds when radiation reaction is neglected. Even if $\Delta_{i}$ is as large as $\left\langle\gamma_{i}\right\rangle / 3$, i.e., the beam has close to $80 \%$ energy spread (full width at half max), the change in $\sigma_{\|}$is at most $5 \%$, and we can safely neglect any effect of the initial energy spread. As an example, we show in Fig. 2 that $\sigma_{\|}$and $\sigma_{\perp}$ are unchanged when the energy spread is increased from 0 to 0.2 of the mean initial energy.

\section{INTENSITY INFERENCE}

\section{A. Plane waves}

We now show that the angular profile and Eq. (4) can be used to obtain the laser intensity, i.e., the collision $a_{0}$, in a model-independent way. The key points are that the analytical result is given in terms of the mean initial and final energies and that the covariance term is negligible. This being the case, $a_{0}$ is fixed by the mean initial and final electron energies, which can be measured; any explicit dependence on radiation-reaction effects is absorbed into the latter quantity. Rearranging Eq. (4), we obtain

$$
a_{0}^{2}=4 \sqrt{2}\left\langle\gamma_{i}\right\rangle\left\langle\gamma_{f}\right\rangle\left(\sigma_{\|}^{2}-\sigma_{\perp}^{2}\right) .
$$

There is no explicit dependence on a classical radiation reaction, because we have taken the product of the final energy and the angular size: While $\gamma_{f} \propto\left(1+R \gamma_{i}\right)^{-1}$, as shown by Eq. (3), $\left(\sigma_{\|}^{2}-\sigma_{\perp}^{2}\right) \propto\left(1+R \gamma_{i}\right)$. Heuristically, because the electron oscillation in an electromagnetic wave is proportional to $a_{0} / \gamma$, a decrease in the energy causes a compensating increase in the size of the electron oscillation and, consequently, the angular spread of the radiation. Furthermore, taking the difference between the parallel and perpendicular variances removes the effect of the electron beam divergence $\delta$, as this term appears in both Eqs. (1) and (2). However, it should be noted that the divergence has an importance not evident in the 1D scenario considered in this section, which is that it controls the expansion of the electron beam and, therefore, its degree of overlap with the laser focal spot. This is discussed in Sec. III B.

Although Eq. (5) is exactly true under a classical radiation reaction (and its absence), we now demonstrate that it works very well under quantum models of radiation reaction as well. We determine what $a_{0}$ must have been for a set of simulations, substituting into Eq. (5) the $\sigma_{\|}, \sigma_{\perp}$, and the mean initial and final electron energies obtained from 
those simulations [29]. We vary the energy spectra of the electron beams, their initial divergence, the laser intensity and duration, and the model of RR used to calculate the dynamics. The laser pulse is modeled as a plane EM wave. In addition to the three models introduced earlier (and described in the Appendix), we also consider a modified classical model, in which radiation losses are continuous, but the strength of the Landau-Lifshitz force is reduced by the Gaunt factor $g(\chi) \leq 1$ [30], and the photon energies are sampled from the quantum synchrotron spectrum. This has been used to describe recent experimental results [11]. It ensures that the power spectrum is quantum corrected but neglects stochastic effects. (See the Appendix for details.) The inferred $a_{0}$ is plotted against the true $a_{0}$ in Fig. 3.

We find that using Eq. (5) to infer the laser $a_{0}$ is accurate to within a few percent across the range of parameters explored, whether the radiation reaction is classical or quantum in nature or absent. If no radiation reaction, or classical RR, is used in the simulations, the agreement is near perfect; when a quantum-corrected model is used instead, the error grows with increasing electron beam energy and $a_{0}$. This suggests that, while $\chi$-dependent corrections can be made to Eqs. (1) and (2), most of their effect is encapsulated in the dependence on the final energy $\gamma_{f}$. For example, in a collision between an electron beam with mean energy $500 \mathrm{MeV}$ (standard deviation $5 \mathrm{MeV}$, orange points in Fig. 3) and a laser pulse with $a_{0}=100$,

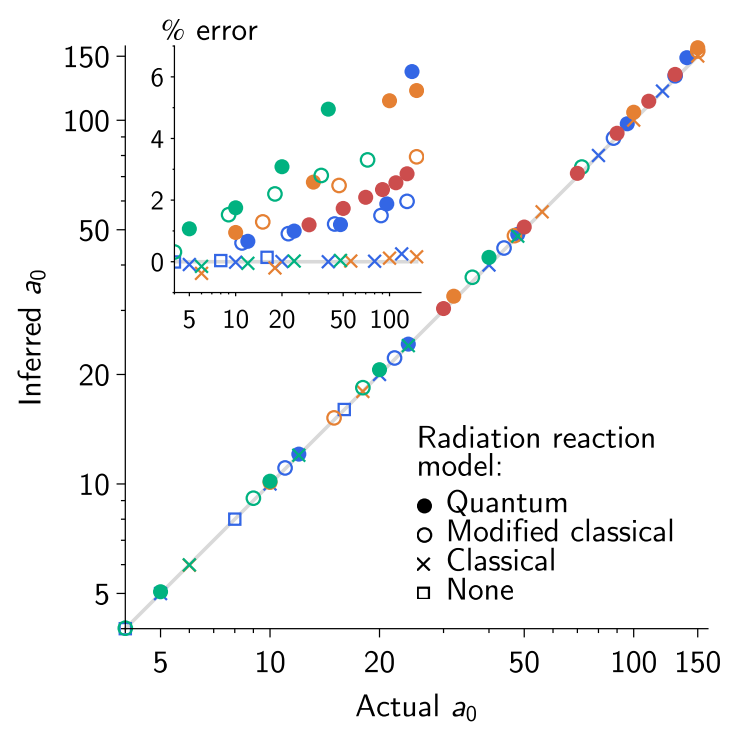

FIG. 3. The $a_{0}$ inferred from simulation results, using Eq. (5), and (inset) the percentage error in the same, for a beam of electrons with normally distributed initial energies (mean $\mu$ and standard deviation $\Delta$ ) and initial divergence $\delta$ colliding with a plane-wave laser pulse with normalized amplitude $a_{0}$, wavelength $0.8 \mu \mathrm{m}$, and FWHM duration $\tau$ : (blue) $\mu=250 \mathrm{MeV}$, $\Delta=5 \mathrm{MeV}, \quad \delta=1 \mathrm{mrad}$, and $\tau=150 \mathrm{fs}$; (orange) $\mu=$ $500 \mathrm{MeV}, \Delta=50 \mathrm{MeV}, \delta=3 \mathrm{mrad}$, and $\tau=20 \mathrm{fs}$; (green) $\mu=1000 \mathrm{MeV}, \Delta=50 \mathrm{MeV}, \delta=2 \mathrm{mrad}$, and $\tau=30 \mathrm{fs}$; and (red) $\mu=200 \mathrm{MeV}, \Delta=10 \mathrm{MeV}, \delta=2 \mathrm{mrad}$, and $\tau=30$ fs. going from the classical to the quantum model increases the mean final energy by a factor of 1.58 but decreases the parallel variance $\sigma_{\|}^{2}$ by a factor of 1.43 . Therefore, the inferred $a_{0}=105$ is close to the actual $a_{0}=100$, which is the classical result.

For $a_{0}>50$, it is advisable to use electron beams of lower energy to minimize the importance of quantum corrections to Eq. (5): Reducing the mean initial energy to 200 (red points in Fig. 3) from $1000 \mathrm{MeV}$ (green points) reduces the error in the inferred $a_{0}$ by more than a factor of 2. The difficulty associated with doing so is that a detector with a larger acceptance angle is required to capture the whole radiation profile, which has characteristic size $\propto a_{0} /\left(\gamma_{i} \gamma_{f}\right)^{1 / 2}$. Our simulation results indicate that capturing all photons with $\theta_{\max } \lesssim 2 a_{0} / \gamma_{f}$ is necessary for the accurate determination of $\sigma_{\|}^{2}$. This angle is almost independent of $\gamma_{i}$ at high $a_{0}$ due to radiative losses, where it grows as $a_{0}^{3}$. These radiative losses also mean that we do not necessarily have $\gamma \gg a_{0}$ throughout the laser pulse, as assumed in our earlier derivation. As such, we expect the method presented here to be limited by the reflection threshold $\gamma_{f} \simeq a_{0}$, above which the reacceleration of the decelerated electrons by the laser pulse becomes significant [31,32]. In fact, quantum effects intercede before this is reached. Nevertheless, the region $5 \lesssim a_{0} \lesssim 150$ shown in Fig. 3 is relevant for a wide variety of laser-electron scattering experiments at existing, and planned, highintensity laser facilities.

In analyzing the simulation results, we have used the fact that the mean initial energy for each individual shot $\left\langle\gamma_{i}\right\rangle$ is known exactly. While the final energy can be measured straightforwardly on a shot-by-shot basis, it is unlikely that this can be done for the initial energy, unless the advanced method proposed by Ref. [33] can be employed. Therefore, the $\left\langle\gamma_{i}\right\rangle$ appearing in Eq. (5) must be obtained from measurements of the electron beam in the absence of the high-intensity laser. However, our results in Fig. 5 indicate that the accuracy of the inferred $a_{0}$ is a few percent, which means, if the stability of the mean initial electron energy is better than $10 \%$, this is not the dominant source of error.

\section{B. Focused fields}

We now consider the application of our results in a more realistic configuration, where we take into account the spatiotemporal structure of a tightly focused laser pulse and the finite size of the electron beam. It is clear that, ideally, the electron beam should be much smaller than the laser focal spot, in the same way that any probe must be smaller than the system to be probed. If this is not the case, the inferred $a_{0}$ will be smaller than the true $a_{0}$, as the radiation profile will have been averaged over the spatial profile of the electron beam. This is still a useful quantity, as it represents an $a_{0}$ that is characteristic of the collision as a whole. Indeed, we show that, if a transverse offset is 
introduced between the electron beam and laser pulse, the reduction in the inferred $a_{0}$ allows the imperfect overlap to be identified.

Consider the collision of an electron beam with a laser pulse that has wavelength $0.8 \mu \mathrm{m}$ and FWHM duration $30 \mathrm{fs}$, which is focused to a spot size of $w_{0}=2 \mu \mathrm{m}$, where $w_{0}$ is the radius at which the intensity falls to $1 / e^{2} \simeq 0.14$ of its peak value. The electron beam has a cylindrically symmetric, Gaussian charge distribution of radius $r_{b}=$ $0.5 \mu \mathrm{m}$ and length $\ell_{b}=5.0 \mu \mathrm{m}$; it has mean energy $750 \mathrm{MeV}$ (standard deviation $100 \mathrm{MeV}$ ), rms divergence $3 \mathrm{mrad}$, and is offset from the laser axis by a perpendicular distance $x_{b}$. The angular distributions of the emitted photons and electron energy spectra for this configuration are shown in Fig. 4, for $a_{0}=30$ and assuming a quantum radiation reaction. The inferred $a_{0}$ are $a_{0}^{\text {inf }}=28.2$ and 16.7 for $x_{b}=0$ and $w_{0}$, respectively. The former is consistent with the true value of $a_{0}$; the reduction in the latter case is evidence that the electron beam has not interacted with the most intense part of the laser pulse. Notice that the average energy loss of the electrons and the angular profile of the radiation are both reduced in size.

We can estimate the reduction due to finite-size effects as follows. An individual electron of the beam, with transverse displacement $x, y$, encounters a peak normalized laser amplitude $a(x, y)=a_{0} \exp \left[-\left(x^{2}+y^{2}\right) / w_{0}^{2}\right]$. As the radiation profile is an integrated signal, the $a_{0}$ inferred from it is $a_{0}^{\text {inf }}=\sqrt{\left\langle a^{4}\right\rangle /\left\langle a^{2}\right\rangle}$ to lowest order in $\alpha$, where the average is taken over the distribution of $x, y$. Then

$$
a_{0}^{\text {inf }}=a_{0} \sqrt{P / Q} \exp \left[-\xi^{2} /(P Q)\right],
$$

where $P=1+4 \rho^{2}, Q=1+8 \rho^{2}, \rho=r_{b} / w_{0}$, and $\xi=x_{b} / w_{0}$. First, we confirm that Eq. (5) provides a modelindependent prediction of the laser $a_{0}$ by repeating the simulations shown in Fig. 4 for different models of radiation reaction: Figure 5 shows that the inferred $a_{0}$ is consistent across all four models tested, at a peak $a_{0}<150$. We also find that the reduction in the inferred $a_{0}$ due to the finite size of the electron beam and the transverse offset is in agreement with Eq. (6).

For the highest $a_{0}$ shown in Fig. 5, the three radiationreaction models, while consistent with each other, separate from the "no RR" result. This is due to ponderomotive scattering, which is the radial expulsion of electrons from a focused field by intensity gradients and, therefore, a source of angular deflection absent in a plane wave. Such deflection is amplified by radiation losses, which reduce $\gamma$ and so the rigidity of the electron beam. Consequently, the radiation profile is broader for a focused field than for a plane wave with the same peak $a_{0}$, and Eq. (5) overestimates the intensity. The error grows to $10 \%$ at $a_{0} \simeq 150$, for a focal spot size $r_{0}=2.0 \mu \mathrm{m}$. Nevertheless, taking this as the upper bound on the validity of the method we have presented, we conclude that it does infer $a_{0}$ in a

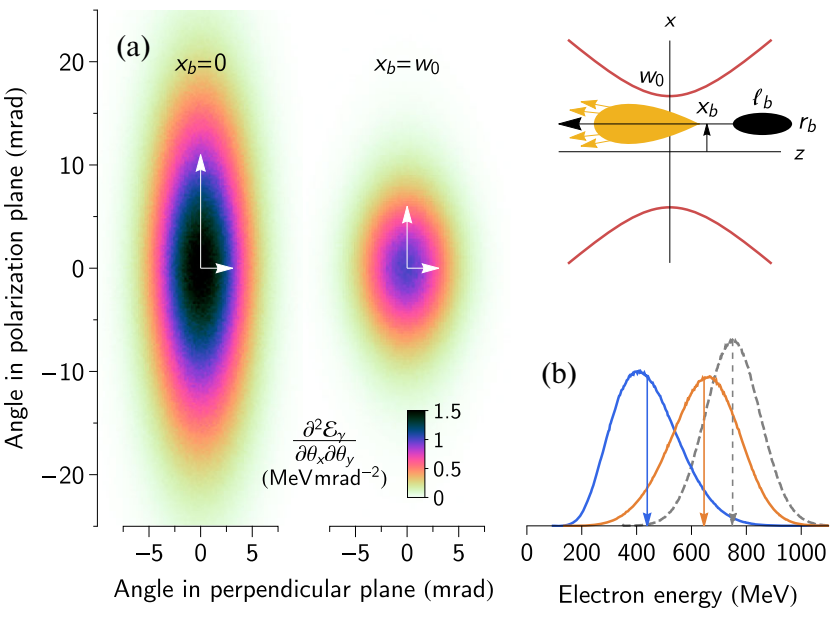

FIG. 4. (a) Energy radiated per unit solid angle (per electron) by an electron beam in a collision with a laser pulse that has peak $a_{0}=30$. White arrows indicate the standard deviations of the distributions. (b) Energy spectra of the electrons before the collision (gray, dashed curve) and after, when the beam offset from the laser axis is $x_{b}=0$ (blue curve) and $x_{b}=w_{0}$ (orange curve). Arrows indicate the mean energy.

model-independent way across approximately 3 orders of magnitude in laser intensity, as shown in Fig. 3.

In the simulations underpinning Figs. 4 and 5, we set the beam radius $r_{b}=0.5 \mu \mathrm{m}$, which corresponds to a full width at half maximum diameter of $1.2 \mu \mathrm{m}$. While beam sizes of this magnitude have been measured in laserwakefield accelerators [34], a large distance between the end of the acceleration stage and the collision point will cause the beam size to be larger. In Fig. 6, we show how the inferred $a_{0}$ depends on the beam radius at fixed $a_{0}=30$, in the cases that there is no transverse offset between the beams $\left(x_{b}=0\right.$, in red) and an offset of $2.0 \mu \mathrm{m}\left(x_{b}=w_{0}\right.$, in blue). We increase the beam radius $r_{b}$ while keeping the divergence $\delta$ fixed, as if the distance between the beam

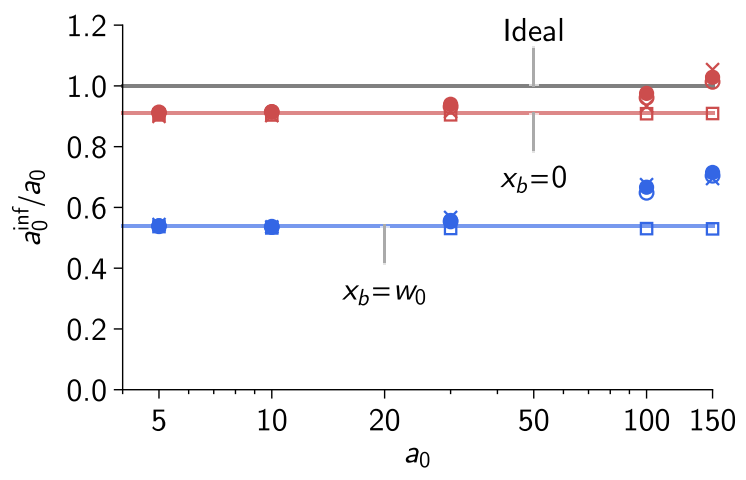

FIG. 5. The inferred $a_{0}$ as a fraction of the true $a_{0}$, for the collision geometry shown in Fig. 4, for different values of $a_{0}$ : from simulations with quantum (filled circles), modified classical (open circles), classical (crosses), and no RR (squares); and as predicted by Eq. (6) for $x_{b}=0$ (red) and $x_{b}=w_{0}$ (blue). 


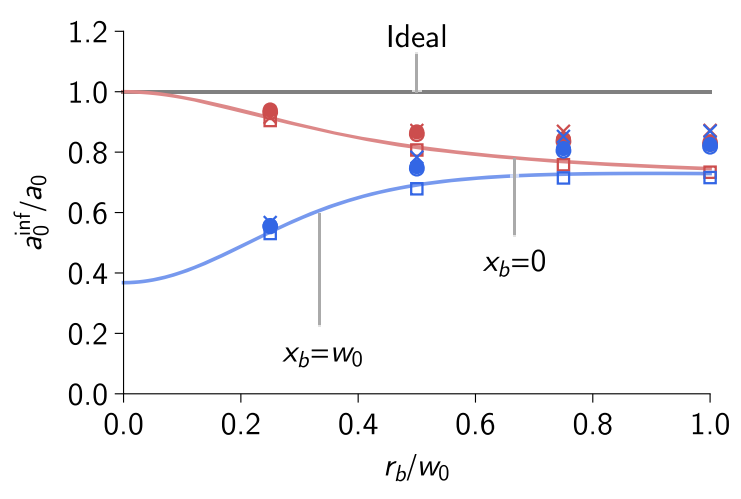

FIG. 6. The inferred $a_{0}$ as a fraction of the true $a_{0}$, for the collision geometry shown in Fig. 4, for different beam radii $r_{b}$ : from simulations with quantum (filled circles), modified classical (open circles), classical (crosses), and no RR (squares); and as predicted by Eq. (6) for $x_{b}=0$ (red) and $x_{b}=w_{0}$ (blue).

generation and collision points had been increased. Our results confirm that increasing the beam size until it is comparable to the size of the laser focal spot has a relatively weak effect on the inferred $a_{0}$. Moreover, it has the additional effect of making the procedure more tolerant of a transverse offset between the beams. The reduction in $a_{0}^{\text {inf }}$ from $a_{0}$ is in good agreement with Eq. (6).

Given a separate estimate, or measurement, of the peak laser intensity, the transverse offset $x_{b}$ could be inferred from the reduction of $a_{0}^{\text {inf }}$ from $a_{0}$. Furthermore, as the overlap between the laser pulse and electron beam varies from shot to shot due to imperfect pointing stability, accumulating the distribution of $a_{0}^{\text {inf }}$ over a large series of collisions could indicate systematic effects such as the finite size of the electron beam.

\section{CONCLUSIONS}

We have shown that, by probing an intense laser pulse with a relativistic electron beam, measuring the angular size of the emitted radiation and the initial and final beam energies, the normalized amplitude $a_{0}$ at the collision point may be inferred in a model-independent way. By "modelindependent," we mean that across 3 orders of magnitude in laser intensity, $5 \lesssim a_{0} \lesssim 150$, different models for the electron dynamics yield a consistent value for the inferred $a_{0}$ that is accurate to within a few percent. As the best choice of model depends on the intensity, relaxing the requirement that one must be assumed a priori means that our method provides strong evidence that a particular intensity range has been reached. This is particularly useful for experiments intended to distinguish between radiationreaction models, as this becomes feasible in a reduced number of successful collisions if $a_{0}$ can be measured independently [35]. The quantities necessary to use Eq. (5), our main result, can be measured on a shot-by-shot basis without additional theoretical modeling. This allows the variation in the collision $a_{0}$ due to shot-to-shot fluctuations to be identified, including the effect of a systematic error in alignment. We emphasize that our method is complementary to model-dependent analysis of the interaction, using, for example, the largest angle of the radiation angular profile [12] or coincidence measurements of the radiation and electron energy spectra [10].

The datasets necessary to reproduce the figures and analysis are available in Ref. [36].

\section{ACKNOWLEDGMENTS}

T. G. B. thanks Arkady Gonoskov for helpful discussions. We acknowledge funding from the Knut and Alice Wallenberg Foundation (T. G. B. and M. M.), the Swedish Research Council (Grant No. 2016-03329, M. M.), and the Engineering and Physical Sciences Research Council (Grant No. EP/M018555/1, S.P. D. M.). This work was supported by the European Research Council (ERC) under the European Union's Horizon 2020 research and innovation program Grant Agreement No. 682399 (S. P. D. M.). Simulations were performed on resources provided by the Swedish National Infrastructure for Computing (SNIC) at the High Performance Computing Centre North (HPC2N).

\section{APPENDIX: MODELS OF RADIATION REACTION AND EMISSION}

We use CIRCE, a particle-tracking code that simulates photon and positron production in prescribed external electromagnetic waves. Collective effects and backreaction are neglected in this framework. There are four possible models of the electron dynamics: no radiation reaction (i.e., Lorentz force only); classical radiation reaction using the Landau-Lifshitz equation; a modified classical model incorporating a quantum correction to the radiated power; and a quantum, stochastic model. Photon emission is handled in the same way for all four models, except that in the former two the photon momentum is sampled from the classical synchrotron spectrum and in the latter two from the quantum synchrotron spectrum. Here, we discuss the four models in detail.

\section{No radiation reaction}

In the no $R R$ model, the electron trajectory follows from the Lorentz force equation:

$$
\dot{p}^{\mu}=-\frac{e F^{\mu \nu} p_{\nu}}{m},
$$

for four-momentum $p$ and field tensor $F$. (Dots denote differentiation with respect to the proper time.) The photon emission rate $\dot{N}_{\gamma}$, differential in photon energy $\omega$, polar angle $\theta$, and azimuthal angle $\varphi$, is controlled by the electron Lorentz factor $\gamma=\left(1-\beta^{2}\right)^{-1 / 2}$, velocity $\beta$, and quantum parameter $\chi=\left|F_{\mu \nu} p^{\nu}\right| /\left(m E_{\mathrm{cr}}\right)$, where $E_{\mathrm{cr}}=m^{2} / e$ is the 
critical field of QED [37]. In the classical limit $\chi \ll 1$, it is given by $[28,38]$ :

$$
\frac{\partial^{3} \dot{N}_{\gamma}}{\partial u \partial z \partial \varphi}=\frac{\alpha m}{3 \sqrt{3} \pi^{2} \chi} u\left(2 z^{2 / 3}-1\right) K_{1 / 3}\left(\frac{2 u z}{3 \chi}\right) .
$$

Here, $u=\omega /(\gamma m), z=\left[2 \gamma^{2}(1-\beta \cos \theta)\right]^{3 / 2}$, and $K$ is a modified Bessel function of the second kind. The domain of Eq. (A2) is $0 \leq u<\infty, 1 \leq z<\infty$, and $0 \leq \varphi<2 \pi$.

The trajectory is obtained numerically using the following methods. If the external field is a plane wave, the particle push takes the following form [27]: The spatial components of the momentum $p^{\mu}$ perpendicular to the laser wave vector $\kappa$ are determined by $\omega_{0} \partial_{\phi} \vec{p}_{\perp}=-e \vec{E}_{\perp}(\phi)$, where $\vec{E}_{\perp}$ is the electric field at phase $\phi$ and the angular frequency $\omega_{0}=\kappa^{0}$. The other two components follow from the conditions $p^{-}=$const and $p^{2}=m^{2}$ and the position from $\omega_{0} \partial_{\phi} x^{\mu}=p^{\mu} / p^{-}$. Here, $p^{-}=\kappa \cdot p / \omega_{0}$ is the light front momentum. If the field is a focused Gaussian beam, and therefore a function of all three spatial coordinates, we use the particle push introduced by Vay [39] and the analytical expressions given in Ref. [40].

To model photon emission, each electron is initialized with an optical depth $T=-\log (1-U)$ for pseudorandom $0 \leq U<1$, which evolves as $\dot{T}=-\dot{N}_{\gamma}$, where $\dot{N}_{\gamma}$ is the instantaneous rate of emission (per unit proper time) obtained by integrating Eq. (A2), until the point where $T$ falls below zero. Then $T$ is reset, and the photon energy $\omega$, polar scattering angle $\theta$, and azimuthal angle $\varphi$ are pseudorandomly sampled from the differential spectrum Eq. (A2).

\section{Classical radiation reaction}

The equation of motion in the classical model is the Landau-Lifshitz equation [18], which adds to Eq. (A1) a continuous radiation-reaction force $f_{\mathrm{LL}}$ that accounts for the loss of energy:

$$
\begin{gathered}
\dot{p}^{\mu}=-\frac{e F^{\mu \nu} p_{\nu}}{m}+f_{\mathrm{LL}}^{\mu}, \\
f_{\mathrm{LL}}^{\mu}=\frac{2 \alpha m}{3}\left[-\frac{F_{, \sigma}^{\mu \nu} p_{\nu} p^{\sigma}}{m^{2} E_{\mathrm{cr}}}+\frac{F^{\mu \nu} F_{\nu \sigma} p^{\sigma}}{E_{\mathrm{cr}}^{2}}-\chi^{2} p^{\mu}\right] .
\end{gathered}
$$

The photon emission rate associated with the resulting trajectory is exactly as given in Eq. (A2).

Numerically, if the external field is a plane wave, the light front momentum, which satisfies $\omega_{0} \partial_{\phi} p^{-}=$ $-(2 \alpha / 3)\left|\vec{E}_{\perp}(\phi) / E_{\mathrm{cr}}\right|^{2} p^{-2}$, is advanced using Heun's method. The remaining components follow from the mass-shell condition and $\omega_{0} \partial_{\phi}\left(\vec{p}_{\perp} / p^{-}\right)=-e \vec{E}_{\perp}(\phi) / p^{-}$, where we have neglected an additional derivative term that is smaller by a factor of $\alpha \omega_{0} / m \ll 1$. If the field is a focused Gaussian beam, we employ the Vay push as before, modified to include RR using the method given in Ref. [41]: The momentum after half a time step is used to calculate the magnitude of the RR force $\vec{f}_{\mathrm{LL}}$, and the associated momentum change $\vec{f}_{\mathrm{LL}} \Delta t$ is added to the momentum change induced by the Lorentz force. We use only the last term in Eq. (A4) to calculate $\vec{f}_{\mathrm{LL}}$, as this is by far the dominant contribution.

Radiation emission is modeled in exactly the same way as in the no RR model. The difference is that the energy radiated, according to Eq. (A2), matches the energy lost by the electron.

\section{Quantum radiation reaction}

In the quantum picture, radiation reaction is the recoil arising from the emission of multiple, incoherent photons. At a high intensity, it is possible to model emission as occurring instantaneously and discretely at stochastically determined points. Thus, the equation of motion between emission events is given by the Lorentz force, Eq. (A1). At an emission event, the electron recoil is determined selfconsistently by the momentum of the photon it has emitted, i.e., $\vec{p} \rightarrow \vec{p}-\vec{k}$.

Therefore, we obtain the trajectory numerically using the same methods as in the no RR case. Photon emission is modeled by sampling the quantum synchrotron spectrum [28]:

$$
\begin{aligned}
\frac{\partial^{3} \dot{N}_{\gamma}}{\partial u \partial z \partial \varphi}= & \frac{\alpha m}{3 \sqrt{3} \pi^{2} \chi} \frac{u}{(1+u)^{3}} \\
& \times\left[z^{2 / 3}\left(2+2 u+u^{2}\right)-(1+u)\right] K_{1 / 3}\left(\frac{2 u z}{3 \chi}\right),
\end{aligned}
$$

where $u=\omega /(\gamma m-\omega), z=\left[2 \gamma^{2}(1-\beta \cos \theta)\right]^{3 / 2}$, and $K$ is a modified Bessel function of the second kind. [The domain of Eq. (A5) is $0 \leq u<\infty, 1 \leq z<\infty$, and $0 \leq \varphi<2 \pi$.] Note that, unlike Eq. (A2), there is no emission of photons with more energy than the electron. As a consequence, the radiated power is reduced, with respect to its classical value, by a factor $g(\chi)$ [30].

As in the no RR and classical models, each electron is initialized with an optical depth $T=-\log (1-U)$ for pseudorandom $0 \leq U<1$, which evolves as $\dot{T}=-\dot{N}_{\gamma}$, where $\dot{N}_{\gamma}$ is the instantaneous probability rate of emission (per unit proper time) obtained by integrating Eq. (A5), until the point where $T$ falls below zero. Then $T$ is reset, and the photon energy $\omega$, polar scattering angle $\theta$, and azimuthal angle $\varphi$ are pseudorandomly sampled from the differential spectrum Eq. (A5). The electron momentum after the emission $p^{\prime}$ is fixed by the conservation of threemomentum, $\vec{p}^{\prime}=\vec{p}-\vec{k}$, which induces an error that is small for ultrarelativistic particles [42]. 


\section{Modified classical radiation reaction}

A well-known deficiency of the classical model is that it predicts the emission of photons with more energy than the electron. This can be corrected phenomenologically by using the quantum emission spectrum Eq. (A5), rather than the classical equivalent Eq. (A2), and weakening the Landau-Lifshitz force Eq. (A4) by the Gaunt factor $g(\chi)$ [30]. Concretely, the equation of motion becomes

$$
\dot{p}^{\mu}=-\frac{e F^{\mu \nu} p_{\nu}}{m}+g(\chi) f_{\mathrm{LL}}^{\mu},
$$

where $f_{\mathrm{LL}}$ is as defined in Eq. (A4). In this way, radiation reaction is still manifest as a continuous force, the energy radiated matches the energy lost, and the first quantum correction is accounted for $[43,44]$. Stochastic effects are, however, lost.

The numerical methods are similar to those employed in the classical case. If the external field is a plane wave, the light front momentum, which satisfies $\omega_{0} \partial_{\phi} p^{-}=$ $-(2 \alpha / 3)\left|\vec{E}_{\perp}(\phi) / E_{\mathrm{cr}}\right|^{2} p^{-2} g(\chi)$, is advanced using Heun's method. The remaining components follow from the massshell condition and $\omega_{0} \partial_{\phi}\left(\vec{p}_{\perp} / p^{-}\right)=-e \vec{E}_{\perp}(\phi) / p^{-}$, where we have neglected an additional derivative term that is smaller by a factor of $\alpha \omega_{0} / m \ll 1$. If the field is a focused Gaussian beam, we employ the Vay push as before, modified to include RR using the method given in Ref. [41]: The momentum after half a time step is used to calculate the magnitude of the RR force $g(\chi) \vec{f}_{\mathrm{LL}}$, and the associated momentum change $g(\chi) \vec{f}_{\mathrm{LL}} \Delta t$ is added to the momentum change induced by the Lorentz force.

Photons are obtained by pseudorandomly sampling the differential spectrum Eq. (A5), as in the quantum case. However, we do not recoil the electron on emission, as the energy loss is already accounted for in the equation of motion.

[1] C. N. Danson et al., Petawatt and exawatt class lasers worldwide, High Power Laser Sci. Eng. 7, e54 (2019).

[2] G. A. Mourou, T. Tajima, and S. V. Bulanov, Optics in the relativistic regime, Rev. Mod. Phys. 78, 309 (2006).

[3] M. Marklund and P. K. Shukla, Nonlinear collective effects in photon-photon and photon-plasma interactions, Rev. Mod. Phys. 78, 591 (2006).

[4] A. Di Piazza, C. Müller, K. Z. Hatsagortsyan, and C. H. Keitel, Extremely high-intensity laser interactions with fundamental quantum systems, Rev. Mod. Phys. 84, 1177 (2012).

[5] N. Neitz and A. Di Piazza, Stochasticity Effects in Quantum Radiation Reaction, Phys. Rev. Lett. 111, 054802 (2013).

[6] T. G. Blackburn, C. P. Ridgers, J. G. Kirk, and A. R. Bell, Quantum Radiation Reaction in Laser-Electron-Beam Collisions, Phys. Rev. Lett. 112, 015001 (2014).
[7] S. Chen, N. D. Powers, I. Ghebregziabher, C. M. Maharjan, C. Liu, G. Golovin, S. Banerjee, J. Zhang, N. Cunningham, A. Moorti, S. Clarke, S. Pozzi, and D. P. Umstadter, MeVEnergy X Rays from Inverse Compton Scattering with Laser-Wakefield Accelerated Electrons, Phys. Rev. Lett. 110, 155003 (2013).

[8] G. Sarri, D. J. Corvan, W. Schumaker, J. M. Cole, A. Di Piazza, H. Ahmed, C. Harvey, C. H. Keitel, K. Krushelnick, S. P. D. Mangles, Z. Najmudin, D. Symes, A. G. R. Thomas, M. Yeung, Z. Zhao, and M. Zepf, Ultrahigh Brilliance Multi-MeV $\gamma$-Ray Beams from Nonlinear Relativistic Thomson Scattering, Phys. Rev. Lett. 113, 224801 (2014).

[9] W. Yan, C. Fruhling, G. Golovin, D. Haden, J. Luo, P. Zhang, B. Zhao, J. Zhang, C. Liu, M. Chen, S. Chen, S. Banerjee, and D. Umstadter, High-order multiphoton Thomson scattering, Nat. Photonics 11, 514 (2017).

[10] J. M. Cole et al., Experimental Evidence of Radiation Reaction in the Collision of a High-Intensity Laser Pulse with a Laser-Wakefield Accelerated Electron Beam, Phys. Rev. X 8, 011020 (2018).

[11] K. Poder et al., Experimental Signatures of the Quantum Nature of Radiation Reaction in the Field of an Ultraintense Laser, Phys. Rev. X 8, 031004 (2018).

[12] O. Har-Shemesh and A. Di Piazza, Peak intensity measurement of relativistic lasers via nonlinear Thomson scattering, Opt. Lett. 37, 1352 (2012).

[13] C. N. Harvey, In situ characterization of ultraintense laser pulses, Phys. Rev. Accel. Beams 21, 114001 (2018).

[14] F. Mackenroth, A. R. Holkundkar, and H.-P. Schlenvoigt, Ultra-intense laser pulse characterization using ponderomotive electron scattering, New J. Phys. 21, 123028 (2020).

[15] M. F. Ciappina, S. V. Popruzhenko, S. V. Bulanov, T. Ditmire, G. Korn, and S. Weber, Progress toward atomic diagnostics of ultrahigh laser intensities, Phys. Rev. A 99, 043405 (2019).

[16] C. Z. He, A. Longman, J. A. Pérez-Hernández, M. de Marco, C. Salgado, G. Zeraouli, G. Gatti, L. Roso, R. Fedosejevs, and W. T. Hill, Towards an in situ, full-power gauge of the focal-volume intensity of petawatt-class lasers, Opt. Express 27, 30020 (2019).

[17] A. Jeandet, A. Borot, K. Nakamura, S. W. Jolly, A. J. Gonsalves, C. Tóth, H.-S. Mao, W. P. Leemans, and F. Quéré, Spatio-temporal structure of a petawatt femtosecond laser beam, J. Phys. Photonics 1, 035001 (2019).

[18] L. D. Landau and E. M. Lifshitz, The Classical Theory of Fields, The Course of Theoretical Physics Vol. 2 (Butterworth-Heinemann, London, 1987).

[19] T. G. Blackburn, D. Seipt, S.S. Bulanov, and M. Marklund, Radiation beaming in the quantum regime, Phys. Rev. A 101, 012505 (2020).

[20] A. Di Piazza, Exact solution of the Landau-Lifshitz equation in a plane wave, Lett. Math. Phys. 83, 305 (2008).

[21] D. Seipt and B. Kämpfer, Asymmetries of azimuthal photon distributions in nonlinear Compton scattering in ultrashort intense laser pulses, Phys. Rev. A 88, 012127 (2013). 
[22] V. I. Ritus, Quantum effects of the interaction of elementary particles with an intense electromagnetic field, J. Sov. Laser Res. 6, 497 (1985).

[23] B. Reville and J. G. Kirk, Computation of synthetic spectra from simulations of relativistic shocks, Astrophys. J. 724, 1283 (2010).

[24] E. Wallin, A. Gonoskov, and M. Marklund, Effects of high energy photon emissions in laser generated ultrarelativistic plasmas: Real-time synchrotron simulations, Phys. Plasmas 22, 033117 (2015).

[25] C. P. Ridgers, J. G. Kirk, R. Duclous, T. G. Blackburn, C. S. Brady, K. Bennett, T. D. Arber, and A. R. Bell, Modelling gamma-ray photon emission and pair production in high-intensity laser-matter interactions, J. Comput. Phys. 260, 273 (2014).

[26] A. Gonoskov, S. Bastrakov, E. Efimenko, A. Ilderton, M. Marklund, I. Meyerov, A. Muraviev, A. Sergeev, I. Surmin, and E. Wallin, Extended particle-in-cell schemes for physics in ultrastrong laser fields: Review and developments, Phys. Rev. E 92, 023305 (2015).

[27] T. G. Blackburn, D. Seipt, S.S. Bulanov, and M. Marklund, Benchmarking semiclassical approaches to strong-field QED: Nonlinear Compton scattering in intense laser pulses, Phys. Plasmas 25, 083108 (2018).

[28] V. N. Baier, V. M. Katkov, and V. M. Strakhovenko, Electromagnetic Processes at High Energies in Oriented Single Crystals (World Scientific, Singapore, 1998).

[29] The mean $\mu$ and standard deviation $\sigma$ of an angular probability density function $p(\theta)$ are defined by the relations $\tan \mu=$ $\langle\sin \theta\rangle /\langle\cos \theta\rangle$ and $\sigma^{2}=-2 \ln \left[\langle\sin \theta\rangle^{2}+\langle\cos \theta\rangle^{2}\right]$, where $\langle f(\theta)\rangle=\int_{0}^{2 \pi} f(\theta) p(\theta) \mathrm{d} \theta$.

[30] T. Erber, High-energy electromagnetic conversion processes in intense magnetic fields, Rev. Mod. Phys. 38, 626 (1966).

[31] J.-X. Li, K. Z. Hatsagortsyan, B. J. Galow, and C. H. Keitel, Attosecond Gamma-Ray Pulses via Nonlinear Compton Scattering in the Radiation-Dominated Regime, Phys. Rev. Lett. 115, 204801 (2015).

[32] M. Vranic, O. Klimo, G. Korn, and S. Weber, Multi-GeV electron-positron beam generation from laser-electron scattering, Sci. Rep. 8, 4702 (2018).
[33] C. D. Baird, C. D. Murphy, T. G. Blackburn, A. Ilderton, S. P. D. Mangles, M. Marklund, and C. P. Ridgers, Realising single-shot measurements of quantum radiation reaction in high-intensity lasers, New J. Phys. 21, 053030 (2019).

[34] M. Schnell, A. Sävert, B. Landgraf, M. Reuter, M. Nicolai, O. Jäckel, C. Peth, T. Thiele, O. Jansen, A. Pukhov, O. Willi, M. C. Kaluza, and C. Spielmann, Deducing the Electron-Beam Diameter in a Laser-Plasma Accelerator Using X-Ray Betatron Radiation, Phys. Rev. Lett. 108, 075001 (2012).

[35] C. Arran, J. M. Cole, E. Gerstmayr, T. G. Blackburn, S. P. D. Mangles, and C. P. Ridgers, Optimal parameters for radiation reaction experiments, Plasma Phys. Controlled Fusion 61, 074009 (2019).

[36] T. G. Blackburn, https://doi.org/10.5281/zenodo.3826949.

[37] J. Schwinger, On gauge invariance and vacuum polarization, Phys. Rev. 82, 664 (1951).

[38] E. Esarey, S. K. Ride, and P. Sprangle, Nonlinear Thomson scattering of intense laser pulses from beams and plasmas, Phys. Rev. E 48, 3003 (1993).

[39] J.-L. Vay, Simulation of beams or plasmas crossing at relativistic velocity, Phys. Plasmas 15, 056701 (2008).

[40] Y. I. Salamin, Fields of a Gaussian beam beyond the paraxial approximation, Appl. Phys. B 86, 319 (2007).

[41] M. Tamburini, F. Pegararo, A. Di Piazza, C. H. Keitel, and A. Macchi, Radiation reaction effects on radiation pressure acceleration, New J. Phys. 12, 123005 (2010).

[42] R. Duclous, J. G. Kirk, and A. R. Bell, Monte Carlo calculations of pair production in high-intensity laserplasma interactions, Plasma Phys. Controlled Fusion 53, 015009 (2011).

[43] C. P. Ridgers, T. G. Blackburn, D. Del Sorbo, L. E. Bradley, C. Slade-Lowther, C. D. Baird, S. P. D. Mangles, P. McKenna, M. Marklund, C. D. Murphy, and A. G. R. Thomas, Signatures of quantum effects on radiation reaction in laser-electron-beam collisions, J. Plasma Phys. 83, 715830502 (2017).

[44] F. Niel, C. Riconda, F. Amiranoff, R. Duclous, and M. Grech, From quantum to classical modeling of radiation reaction: A focus on stochasticity effects, Phys. Rev. E 97, 043209 (2018). 